WINPEC Working Paper Series No.E2015

October 2020

Stable Coalition Structures and Power Indices for Majority Voting Takaaki Abe

Waseda I Nstitute of Political EConomy Waseda University

Tokyo, J apan 


\title{
Stable Coalition Structures and Power Indices for Majority Voting
}

\author{
Takaaki Abe*
}

October 1, 2020

\begin{abstract}
An (n,k)-game is a voting game in which each player has exactly one vote, and decisions are made by at least $\mathrm{k}$ affirmative votes of the $\mathrm{n}$ players. A power index is a measure of the a priori power of the $\mathrm{n}$ voters. The purpose of this paper is to show what axioms of power indices generate stable coalition structures for each $(\mathrm{n}, \mathrm{k})$-game. Using the stability notion of the core, we show that a coalition structure containing a minimal winning coalition is stable for a wide range of general power indices satisfying a set of axioms, such as the Shapley-Shubik, Banzhaf, normalized Banzhaf, and Deegan-Packel power indices. Moreover, we also show that a coalition structure that represents a two-party system can be stable if the two large parties are close enough in size. Some unstable coalition structures are also analyzed.
\end{abstract}

Keywords: coalition structure; core; majority voting; power index

JEL Classification: C71

\section{Introduction}

An $(n, k)$-game should be one of the most familiar voting systems. For example, for a constitutional amendment to pass, the amendment must be supported by a majority of the voters, and each country adopts its own quota $k, e . g ., 1 / 2+, 3 / 5,2 / 3$ and $3 / 4$, which defines the size of the majority of the voting. In an $(n, k)$-game, voters are symmetric in the sense of one person, one vote. However, when voters form coalitions or political parties, the "power" of voters can be asymmetric: the power of a voter who belongs to a large party may differ from that of a member of a small party. In the theory of cooperative games, the notion of power has been called voting power, and various power indices have been proposed. The most famous power index is likely the Shapley-Shubik power index proposed by Shapley and Shubik (1954). In addition, the Banzhaf power index and the Deegan-Packel power index were proposed by Banzhaf (1965) and Deegan and Packel (1978), respectively. However, these power index notions do not take into account the coalition structure of voters such as political parties. Owen (1977) assumed that

* School of Political Science and Economics, Waseda University. 1-6-1, Nishi-waseda, Shinjuku-ku, Tokyo 169-8050, Japan. Email: takatomo3639@asagi.waseda.jp

The author appreciates the helpful comments provided by Yukio Koriyama and Shigeo Muto. The author gratefully acknowledges the financial support from JSPS Grant-in-Aid for Research Activity Start-up (No.19K23206) and Waseda Univeristy Grant-in-Aid for Research Base Creation (2019C-486). 
people form a coalition structure for economic and political reasons and extended the Shapley-Shubik power index to cooperative games with coalition structures.

Hart and Kurz (1984) utilized the Owen power index and analyzed stable coalition structures in an $(n, k)$-game. They assumed that players deviate from a coalition structure to improve their voting power. They used the $\alpha-, \beta-, \gamma$ - and $\delta$-stability concepts that were proposed in their preceding paper Hart and Kurz (1983). Abe (2018) also uses the Owen power index to study stable coalition structures in a symmetric majority game. Instead of the preceding four stability notions, he uses the pessimistic core and the projective core studied by Funaki and Yamato (1999), Bloch and van den Nouweland (2014) and Abe and Funaki (2017).*1

In this paper, we no longer restrict power indices to the Owen power index and do not use any specific power index. We generalize a power index to some axioms and study which axiomatic properties generate stable coalition structures. Therefore, our results apply to every power index as long as the power index satisfies the axioms required in our propositions. The motivation for this generalization lies in the HartKurz assumption mentioned above: players deviate from a coalition structure to improve their voting power. This assumption suggests that a power index represents players' incentive to change coalition structures. Therefore, choosing a specific power index may yield results that hold only for the chosen power index and may conceal the axioms that are common to several power indices and contribute to stable coalition structures.

Moreover, we also generalize the method for calculating voting power: in the papers mentioned above, the voting power of a single player in a coalition is simply given by "the coalitional voting power / the number of members of the coalition" due to the principle of one person, one vote. However, Penrose (1946) argued that the voting power of each member of a coalition should be given by "the coalitional voting power / the square root of the number of members." Therefore, in this paper, we generalize these methods and employ "the coalitional voting power / the number of members to the power of $\delta$ " for $0<\delta \leq 1$. If $\delta=1$, it corresponds to the method of Owen, and if $\delta=0.5$, it fits that of Penrose.

Our generalized definition of voting power is elaborated in Section 2. The core notions are also introduced in Section 2. Section 3 is devoted to introducing the axioms of power indices. Section 4 consists of two parts: in the first part, we study the pessimistic core; in the second part, we address the projective core. In Section 5, we provide some concluding remarks. The proofs are provided in the appendix.

\section{Preliminaries}

\subsection{Voting games and power indices}

For each natural number $n$, let $N=\{1, \ldots, n\}$ be a set of players. Let $S \subseteq N$ be a coalition of players. Let $|S|$ denote the number of players in coalition $S$. We sometimes write $s:=|S|$ for simplicity. Let

\footnotetext{
*1 The notion of farsighted vNM stability, initially proposed by Chwe (1994) and Xue (1997), is also used. In addition to the farsighted vNM stability and the core notions, Kóczy (2007) proposes a different farsighted core concept, called the recursive core. This core concept assumes that deviating players recursively consider the reaction of residual players.
} 
$v: 2^{N} \rightarrow\{0,1\}$ with $v(\emptyset)=0$ and $v(N)=1$ be a simple game. Let $\mathrm{SG}_{N}$ denote the set of simple games with player set $N$. Coalitions with worth 1 are called winning, and coalitions with worth 0 are called losing. A winning coalition for which every proper subset is losing is called minimal winning coalition. For every simple game $v$, let $\mathcal{W}_{v}$ be the set of winning coalitions and $\mathcal{W}_{v}^{\text {min }}$ be the set of minimal winning coalitions. Player $i \in N$ is said to be the dictator in $v$ if $S \in \mathcal{W}_{v} \Longleftrightarrow i \in S$. Player $i \in N$ is said to be a veto player in $v$ if $i \in \cap_{S \in \mathcal{W}_{v}} S$. Player $i \in N$ is said to be a null player in $v$ if $v(S \cup\{i\})-v(S)=0$ for every $S \subseteq N \backslash\{i\}$. A simple game is said to be an $(n, k)$-game if $v(S)=1 \Longleftrightarrow s \geq k$, where $k$ means the minimal number of people needed to win. In many actual voting systems, $k$ is chosen to be over half. We use $H(n)$ to denote the number of over half of people: formally, for every $n \geq 3$,

$$
H(n)= \begin{cases}\frac{n}{2}+1 & \text { if } n \text { is even, } \\ \frac{n+1}{2} & \text { if } n \text { is odd. }\end{cases}
$$

A power index $\psi$ is a function that assigns a vector to each simple game, $\psi: \mathrm{SG}_{N} \rightarrow[0,1]^{N}$. The vector assigned to a game is interpreted as a power distribution over the $n$ players in the voting game. Below, we introduce some voting power indices. The axiomatic characterizations of the Shapley-Shubik, Banzhaf and normalized Banzhaf power indices are studied and compared by van den Brink and van der Laan (1998). Deegan and Packel (1978) provide the axiomatic characterization of the Deegan-Packel power index in their own paper.

- The Shapley-Shubik power index: Let $\sigma$ be an ordering of players in $N$. Player $i$ is the $\sigma(i)$-th player in ordering $\sigma$. Let $\mathrm{PR}_{i}^{\sigma}=\{j \in N \mid \sigma(j)<\sigma(i)\}$ be the set of predecessors of player $i$ in $\sigma$. Player $i$ is called the pivot of $\sigma$ in a simple game $v$ if $\operatorname{PR}_{i}^{\sigma} \notin \mathcal{W}_{v}$ and $\operatorname{PR}_{i}^{\sigma} \cup\{i\} \in \mathcal{W}_{v}$. The Shapely-Shubik power index is given as follows:

$$
\operatorname{SS}_{i}(v)=\frac{\mid\{\sigma \mid i \text { is the pivot of } \sigma\} \mid}{n !} .
$$

- The Banzhaf power index: The Banzhaf power index aggregates the number of swings for each player: a swing for player $i$ is coalition $S$ such that $i \in S, S \in \mathcal{W}_{v}$ and $S \backslash\{i\} \notin \mathcal{W}_{v}$. The Banzhaf power index for player $i$ is defined as the number of swings for $i$ divided by the number of coalitions containing $i$, namely,

$$
\mathrm{BZ}_{i}(v)=\frac{\mid\{S \subseteq N \mid S \text { is a swing for } i\} \mid}{2^{n-1}} .
$$

- The normalized Banzhaf power index: Since the Banzhaf power index does not satisfy efficiency, the index is normalized to sum to one. Note that this power index is also known as the BanzhafColeman power index.

$$
\mathrm{NBZ}_{i}(v)=\frac{\mathrm{BZ}_{i}(v)}{\sum_{j \in N} \mathrm{BZ}_{j}(v)} .
$$

- The Deegan-Packel power index: The Deegan-Packel power index is defined based on minimal winning coalitions. The index aggregates player $i$ 's share, $1 /|S|$, for every minimal winning coalition $S$ that contains $i$ as its member:

$$
\operatorname{DP}_{i}(v)=\frac{1}{\left|\mathcal{W}^{\min }\right|} \sum_{\substack{S \in \mathcal{W}^{\min } \\ i \in S}} \frac{1}{|S|} .
$$




\subsection{Extending a power index to games with coalition structures}

The power indices mentioned above are defined for "normal" simple games, namely, games without coalition structures. Owen (1977) assumed that people form groups and teams and generate a coalition structure, such as political parties, and extended the Shapley-Shubik power index to games with coalition structures. Below, we extend a general power index, that is not restricted to the Shapley-Shubik power index, to games with coalition structures in a similar manner to Owen (1977).

Let $\mathcal{P}$ denote a partition or a coalition structure. These two terms are synonyms in this paper. For every nonempty $S \subseteq N$, let $\Pi(S)$ be the set of partitions of $S$. By $|\mathcal{P}|$, we denote the number of coalitions in $\mathcal{P}$, and by $\mathcal{P}(i)$, we denote the coalition to which player $i$ belongs. Now, let $n \geq 3, H(n) \leq k \leq n$ and $\mathcal{P} \in \Pi(N)$. Moreover, let $\mathcal{P}=\left\{S_{1}, \ldots, S_{m}\right\}$, where $m=|\mathcal{P}|$. Let $M=\{1, \ldots, m\}$. For the given $(n, k)$ and $\mathcal{P}$, define a weighted majority game $w^{(n, k), \mathcal{P}}$ as follows:

$$
w^{(n, k), \mathcal{P}}=\left(k ;\left|S_{1}\right|, \ldots,\left|S_{m}\right|\right),
$$

where for every $R \subseteq M$,

$$
w^{(n, k), \mathcal{P}}(R)= \begin{cases}1 & \text { if } \sum_{j \in R}\left|S_{j}\right| \geq k \\ 0 & \text { otherwise }\end{cases}
$$

In game $w^{(n, k), \mathcal{P}}$, each coalition $S \in \mathcal{P}$ is a player, and $\mathcal{P}$ is its player set. Note that coalition $S$ has $|S|$ votes in the weighted majority game $w^{(n, k), \mathcal{P}}$. A union $R$ of coalitions wins if its total votes reaches the quota $k$. Now, for each $n$ and $k$, the number given as

$$
d:=n-k+1,
$$

plays an important role: $d$ is the minimal number of votes to form a blocking coalition in the $(n, k)$-game. Therefore, for every $\mathcal{P} \in \Pi(N)$, if a coalition $S \in \mathcal{P}$ has $d$ or more members, the coalition $S$ is a veto player in $w^{(n, k), \mathcal{P}}$.

Since $w^{(n, k), \mathcal{P}}$ is a simple game, we can apply a power index $\psi$ to $w^{(n, k), \mathcal{P}}$. The power index $\psi$ assigns voting power $\psi_{S}\left(w^{(n, k), \mathcal{P}}\right)$ to each coalition $S \in \mathcal{P}$, where $\psi_{S}\left(w^{(n, k), \mathcal{P}}\right)$ stands for the coalitional voting power of $S$. Since every member of coalition $S$ has exactly one vote, they are equal within $S$. Hence, let $\frac{1}{|S|} \psi_{S}\left(w^{(n, k), \mathcal{P}}\right)$ denote the power per capita for each player in coalition $S$. If one employs the Shapley-Shubik power index as $\psi$, then the corresponding power index per capita is called the Owen power index.

In Owen's approach described above, we simply divided the coalitional voting power by the number of members of the coalition. On the other hand, Penrose (1946) argued that the voting power of each member is given by $\frac{1}{\sqrt{|S|}} \psi_{S}$ instead of $\frac{1}{|S|} \psi_{S}$, commonly known as Penrose's square-root law. In this paper, we incorporate both approaches: we define individual voting power as $\frac{1}{|S|^{\delta}} \psi_{S}$ for $0<\delta \leq 1$.

Summarizing the steps discussed above, for every $(n, k)$-game, every power index $\psi$ and every $\delta$ with $0<\delta \leq 1$, player $i$ 's voting power in partition $\mathcal{P} \in \Pi(N)$ is defined as follows: for every $i \in N$,

$$
\phi_{i}^{\psi,(n, k), \delta}(\mathcal{P}):=\frac{1}{|\mathcal{P}(i)|^{\delta}} \psi_{\mathcal{P}(i)}\left(w^{(n, k), \mathcal{P}}\right) .
$$




\subsection{Stability notions}

Now, we define the stability of coalition structures. In the same manner as Hart and Kurz (1984), we assume that players deviate from a coalition structure to improve their voting power. In this subsection, we arbitrarily fix $n, k, \delta$ and $\psi$.

We first introduce the projective core. For every nonempty $S \subseteq N$, let $\mathcal{P}_{S}=\{C \cap S \mid C \in \mathcal{P}, C \cap S \neq$ $\emptyset\} \in \Pi(S)$ be the projection of partition $\mathcal{P}$ onto $S$. A coalition $S \subseteq N$ projectively deviates from $\mathcal{P} \in \Pi(N)$ if $\phi_{j}^{\psi,(n, k), \delta}\left(\{S\} \cup \mathcal{P}_{N \backslash S}\right)>\phi_{j}^{\psi,(n, k), \delta}(\mathcal{P})$ for every $j \in S$. A partition $\mathcal{P}$ is said to be projectively stable if no coalition projectively deviates from $\mathcal{P}$. The projective core, $C^{\operatorname{proj}}\left(\phi^{\psi,(n, k), \delta}\right)$, is the set of projectively stable partitions. When coalition $S$ projectively deviates from coalition structure $\mathcal{P}$, the coalition structure $\mathcal{P}_{N \backslash S}$ of the non-deviating players is left unchanged after the deviation, and the resulting coalition structure is $\{S\} \cup \mathcal{P}_{N \backslash S}$. This definition is compatible with the notion of strong Nash equilibrium in the sense that the non-deviating players are assumed not to change their move or coalition structure. In addition, deviating players might be interpreted as myopic players because when they decide to deviate, they consider the coalition structure that results immediately from the deviation and do not anticipate any reorganization of the coalition structure of the other players. The following concept differs from the projective core in this aspect.

A coalition $S \subseteq N$ pessimistically deviates from $\mathcal{P} \in \Pi(N)$ if $\phi_{j}^{\psi,(n, k), \delta}(\{S\} \cup \mathcal{Q})>\phi_{j}^{\psi,(n, k), \delta}(\mathcal{P})$ for every $j \in S$ and every $\mathcal{Q} \in \Pi(N \backslash S)$. A partition $\mathcal{P}$ is said to be pessimistically stable if no coalition pessimistically deviates from $\mathcal{P}$. The pessimistic core, $C^{\text {pes }}\left(\phi^{\psi,(n, k), \delta}\right)$, is the set of pessimistically stable partitions. When some players pessimistically deviate from a coalition structure, they anticipate a worstcase scenario in which their deviation may lead to the following: the other players may "punish" the deviating players and decrease the latter's voting power by reorganizing the coalition structure of nondeviating players. In this sense, this concept presumes careful players and is compatible with the $\alpha$-core proposed by Hart and Kurz (1983). Note that the projective core is a subset of the pessimistic core.

\section{Axioms of power indices}

This section is devoted to introducing general axioms of power indices. Let $N$ be an arbitrary player set and $v$ be a simple game with player set $N{ }^{* 2}$ Let $V T_{v} \subseteq N$ be the set of veto players and $N L_{v} \subseteq N$ be the set of null players in $v$. A simple game $v$ is said to be a veto or null voting game (a vn-game) if $V T_{v} \cup N L_{v}=N$. Let $\mathrm{VNG}_{N}$ denote the set of vn-games with player set $N$.

Below, we introduce some axioms of power indices $\psi: \mathrm{SG}_{N} \rightarrow[0,1]^{N}$. Table 1 shows which of the four power indices listed in the preceding section satisfies the following axioms. The first four axioms describe power distributions for special players such as dictators, veto players and null players.

Axiom 1. (Dictator Property, D)

\footnotetext{
*2 As elaborated in Section 2, a power index is applied to the weighted majority game $w^{(n, k), \mathcal{P}}$, in which players are coalitions in $\mathcal{P}$. Therefore, the player set " $N$ " used in this section refers to the player set of game $w^{(n, k), \mathcal{P}}$, that is, $\mathcal{P}$. Therefore, we do not assume $n \geq 3$ in this section. Let $n \geq 1$.
} 
For every $v \in \mathrm{SG}_{N}$, if $i \in N$ is the dictator in $v$, then $\psi_{i}(v)=1$ and $\psi_{j}(v)=0$ for every $j \in N \backslash\{i\}$.

Axiom 2. (Equal Treatment Property for vn-Voting, ET\#)

For every $v \in \mathrm{VNG}_{N}, \psi_{i}(v)=\psi_{j}(v)$ for every $i, j \in V T_{v}$.

Axiom 3. (Equal Treatment Property for Unanimous Voting, ET-)

For every $v \in \mathrm{SG}_{N}$, if $\mathcal{W}_{v}=\{N\}$, then $\psi_{i}(v)=\psi_{j}(v)$ for every $i, j \in N$.

Axiom 4. (Null Player Property for vn-Voting, NP\#)

For every $v \in \mathrm{VNG}_{N}, \psi_{j}(v)=0$ for every $j \in N L_{v}$.

The four power indices all satisfy these four axioms. Note that ET\# $\Rightarrow$ ET- because $\mathcal{W}_{v}=\{N\}$, which means that all players are veto players, is equivalent to $V T_{v}=N$ and implies that $V T_{v} \cup N L_{v}=N$. In the same manner, the following three efficiency axioms obey $\mathbf{E F} \# \Rightarrow \mathbf{E F}-\Rightarrow \mathbf{E F} 2-$.

Axiom 5. (Efficiency for vn-Voting, EF\#)

For every $v \in \mathrm{VNG}_{N}$, then $\sum_{j \in N} \psi_{j}(v)=1$.

Axiom 6. (Efficiency for Unanimous Voting, EF-)

For every $v \in \mathrm{SG}_{N}$, if $\mathcal{W}_{v}=\{N\}$, then $\sum_{j \in N} \psi_{j}(v)=1$.

Axiom 7. (Efficiency for Two-person Unanimous Voting, EF2-)

For every $v \in \mathrm{SG}_{N}$, if $N=\{i, j\}, i \neq j$ and $\mathcal{W}_{v}=\{N\}$, then $\psi_{i}(v)+\psi_{j}(v)=1$.

The Banzhaf power index violates efficiency in general. The index violates even EF-. For example, consider the following three-person weighted majority game with quota $6:(6 ; 3,2,2)$, where the grand coalition of all three players, which has seven votes, is the only winning coalition. We have $\mathrm{BZ}_{1}=\mathrm{BZ}_{2}=$ $\mathrm{BZ}_{3}=\frac{1}{4}$, and their sum is less than one. However, fortunately, the Banzhaf power index satisfies EF2-, which is less demanding than EF-.

Axiom 8. (Feasibility, F)

For every $v \in \mathrm{SG}_{N}, \sum_{j \in N} \psi_{j}(v) \leq 1$.

Axiom 9. (Feasibility for Unanimous Voting, F-)

For every $v \in \mathrm{SG}_{N}$, if $\mathcal{W}_{v}=\{N\}$, then $\sum_{j \in N} \psi_{j}(v) \leq 1$.

The Banzhaf power index also does not satisfy feasibility: its sum exceeds one for some games. For example, consider $(6 ; 4,1,1,1)$. We have $\mathrm{BZ}_{1}=\frac{1}{2}$ and $\mathrm{BZ}_{2}=\mathrm{BZ}_{3}=\mathrm{BZ}_{4}=\frac{1}{4}$. However, the index satisfies F-.

\section{Stable coalition structures}

\subsection{The pessimistic core}

In this section, we provide our stability results. As mentioned in the introduction, we show what axioms of power indices contribute to stable coalition structures for each $(n, k)$-game and each discount 


\begin{tabular}{llllllllll} 
& D & ET\# & ET- & NP\# & EF\# & EF- & EF2- & F & F- \\
\hline $\mathrm{SS}$ & + & + & + & + & + & + & + & + & + \\
$\mathrm{BZ}$ & + & + & + & + & - & - & + & - & + \\
$\mathrm{NBZ}$ & + & + & + & + & + & + & + & + & + \\
$\mathrm{DP}$ & + & + & + & + & + & + & + & + & + \\
\hline
\end{tabular}

Table 1 Axioms and power indices

factor $\delta$. We assume that $n \geq 3$. The first result shows that the pessimistic core is nonempty for every quota $k$ between $H(n)$ and $n$ and every $\delta$ between 0 and 1 . Nonemptiness is supported by the following three axioms.

Proposition 4.1. Let $H(n) \leq k \leq n$ and $0<\delta \leq 1$. If $\psi$ satisfies $\mathbf{D}$, ET-, F-, then $C^{\text {pes }}\left(\phi^{\psi,(n, k), \delta}\right)$ is nonempty.

This proposition holds for every power index as long as it satisfies the three axioms, and as Table 1 shows, the four power indices all satisfy these axioms. Moreover, nonemptiness holds for every quota $k$ more than $50 \%$ and every possible $\delta$.

Now, what coalition structure belongs to the pessimistic core? As elaborated in the proof, if a coalition structure contains a minimal winning coalition, namely, a $k$-player coalition, then the coalition structure is stable in the sense of the pessimistic core. However, if a partition contains a non-minimal winning coalition, namely, a coalition that consists of more than $k$ players, then it does not lie in the pessimistic core because a minimal winning coalition deviates and wins "more efficiently" with fewer members than the non-minimal winning coalition. Therefore, containing a minimal winning coalition is a sufficient condition for a partition to be in the pessimistic core.

However, it is not a necessary condition: some partitions without any winning coalitions can belong to the pessimistic core. For example, let $(n, k)=(6,5), \delta=1$ and $\psi=$ SS. Table 2 shows the distributions of voting power for these parameters, in which we use a simplified notation: for example, $[4,1,1]$ stands for a partition with one four-person coalition and two one-person coalitions. Partition $[3,2,1]$, in which every coalition consists of fewer than $k(=5)$ players, belongs to the pessimistic core. Why do even coalition structures without any winning coalition satisfy the stability of the pessimistic core? Below, we show that a "two-party system" plays a key role in answering this question.

Recall that $d:=n-k+1$. Let $V T(\mathcal{P}):=\{S \in \mathcal{P}|| S \mid \geq d\}$ for every $\mathcal{P} \in \Pi(N)$. In other words, $V T(\mathcal{P})$ is the set of blocking coalitions in $\mathcal{P}$. This is equivalent to the set of veto players in the weighted majority game $w^{(n, k), \mathcal{P}}$ since each coalition is a player. Moreover, let $S_{\mathcal{P}}^{\min }:=\arg \min _{S \in V T(\mathcal{P})}|S|$, $S_{\mathcal{P}}^{\max }:=\arg \max _{S \in V T(\mathcal{P})}|S|$, and $b_{\mathcal{P}}:=\left|S_{\mathcal{P}}^{\max }\right|-\left|S_{\mathcal{P}}^{\min }\right|$. Therefore, $b_{\mathcal{P}}$ stands for the difference in size between the largest and smallest blocking coalitions. We define integer $a^{*}$ as the remainder of $n / d$, $0 \leq a^{*} \leq n-k$. Now, for every $\delta$ with $0<\delta \leq 1$, define

$$
\Lambda^{n, k}(\delta)=\min \left\{(k-1) \sqrt[\delta]{2}, d \sqrt[\delta]{\frac{n-a^{*}}{d}}\right\} .
$$




\begin{tabular}{|c|c|c|c|c|c|c|c|}
\hline \multicolumn{2}{|l|}{ Partition } & \multicolumn{5}{|c|}{ Voting power per capita $\phi$} & \multirow[t]{2}{*}{$C^{\text {pes }}$} \\
\hline$[6]$ & $1 / 6$ & $1 / 6$ & $1 / 6$ & $1 / 6$ & $1 / 6$ & $1 / 6$ & \\
\hline$[5,1]$ & $1 / 5$ & $1 / 5$ & $1 / 5$ & $1 / 5$ & $1 / 5$ & 0 & + \\
\hline$[4,2]$ & $1 / 8$ & $1 / 8$ & $1 / 8$ & $1 / 8$ & $1 / 4$ & $1 / 4$ & \\
\hline$[4,1,1]$ & $1 / 6$ & $1 / 6$ & $1 / 6$ & $1 / 6$ & $1 / 6$ & $1 / 6$ & \\
\hline$[3,3]$ & $1 / 6$ & $1 / 6$ & $1 / 6$ & $1 / 6$ & $1 / 6$ & $1 / 6$ & \\
\hline$[3,2,1]$ & $1 / 6$ & $1 / 6$ & $1 / 6$ & $1 / 4$ & $1 / 4$ & 0 & + \\
\hline$[3,1,1,1]$ & $1 / 6$ & $1 / 6$ & $1 / 6$ & $1 / 6$ & $1 / 6$ & $1 / 6$ & \\
\hline$[2,2,2]$ & $1 / 6$ & $1 / 6$ & $1 / 6$ & $1 / 6$ & $1 / 6$ & $1 / 6$ & \\
\hline$[2,2,1,1]$ & $5 / 24$ & $5 / 24$ & $5 / 24$ & $5 / 24$ & $1 / 12$ & $1 / 12$ & \\
\hline$[2,1,1,1,1]$ & $1 / 5$ & $1 / 5$ & $3 / 20$ & $3 / 20$ & $3 / 20$ & $3 / 20$ & \\
\hline$[1,1,1,1,1,1]$ & $1 / 6$ & $1 / 6$ & $1 / 6$ & $1 / 6$ & $1 / 6$ & $1 / 6$ & \\
\hline
\end{tabular}

Table $2 \quad(n, k)=(6,5), \delta=1, \psi=\mathrm{SS}$

Function $\Lambda$ consists of the following components. For the left element of the minimization, term $(k-1)$ means the size of that largest nondictatorial blocking coalition, and for the right element, term $d$ stands for the size of the smallest blocking coalition. Moreover, the terms with $\sqrt[\delta]{ }$, namely, 2 and $\left(n-a^{*}\right) / d$, mean the number of blocking coalitions in $\mathcal{P}$. In particular, $\left(n-a^{*}\right) / d$ describes how many $d$-person coalitions can coexist simultaneously for $n$. Note that $\left(n-a^{*}\right) / d$ is an integer and is more than or equal to $|V T(\mathcal{P})|$ for every $\mathcal{P} \in \Pi(N)$.

The following proposition describes the stability of a two-party system and its generalization. The key condition of Proposition 4.2 is (ii), which states that if the difference $b_{\mathcal{P}}$ between the largest and smallest blocking coalitions is small enough, then the partition can be in the pessimistic core.

Proposition 4.2. Let $H(n) \leq k \leq n, 0<\delta \leq 1$ and $\mathcal{P} \in \Pi(N)$. Let $\psi$ satisfy EF\#, ET\#, NP\#. If $\mathcal{P}$ and $\delta$ satisfy the following conditions, then $\mathcal{P} \in C^{\text {pes }}\left(\phi^{\psi,(n, k), \delta}\right)$ :

(i) $\sum_{S \in V T(\mathcal{P})}|S|=k$,

(ii) $b_{\mathcal{P}} \leq \frac{1}{\sqrt[\delta]{|V T(\mathcal{P})|}} \Lambda^{n, k}(\delta)-\left|S_{\mathcal{P}}^{\min }\right|$.

(iii) $\delta \geq \frac{\log (|V T(\mathcal{P})|)}{\log \left(k /\left|S_{\mathcal{P}}^{\min }\right|\right)}$

Note that if the number of blocking coalitions in $\mathcal{P}$ is exactly one, namely, $|V T(\mathcal{P})|=1$, then Proposition 4.2 is a special case of Proposition 4.1 because (i) implies that $\left|S_{\mathcal{P}}^{\min }\right|=k$ and (iii) implies that $\delta=1$; hence, (ii) always holds due to $k \geq 2$ for $n \geq 3$. Therefore, we consider $\mathcal{P}$ with $|V T(\mathcal{P})| \geq 2$ below.

Partition $\mathcal{P}$ with $|V T(\mathcal{P})|=2$ can be regarded as a two-party system, in which blocking coalitions $S_{1}$ and $S_{2} \in V T(\mathcal{P})$ are two competing large parties, and each of them is large enough for the other not to be dictatorial. Condition (i) suggests that coalitions other than the two large parties in the partition are minor parties or independents. To be more precise, (i) implies that each coalition $S \in \mathcal{P} \backslash V T(\mathcal{P})$ is a null player in $w^{(n, k), \mathcal{P}}$ : the power of those minor coalitions is so small that they do not influence any decision 
made in the coalition structure. One might consider that (i) can be replaced by $\sum_{S \in V T(\mathcal{P})}|S| \geq k$. However, if $\sum_{S \in V T(\mathcal{P})}|S|>k$, then (i) no longer implies that coalition $S \in \mathcal{P} \backslash V T(\mathcal{P})$ is a null player in $w^{(n, k), \mathcal{P}}$. For example, if $n=9, k=6$ and $\mathcal{P}=[4,3,2]$, then we have $4+3>6$, while the two-person coalition is not a null player in $w^{(n, k), \mathcal{P}}$. Therefore, (i) is an appropriate condition to describe a coalition structure in which minor parties are not influential.

Condition (ii) suggests that if the two large parties are close in size, then the structure is stable in the sense of the pessimistic core. An intuition of this result is given as follows: players inside the two large parties have strong power and do not have an incentive to change the current structure, while those outside the large parties have an incentive to change the structure but cannot realize it because (a) if the outside players form a deviating coalition that consists only of outside players, then the large parties remain intact, and the outside players cannot improve their voting power; (b) if they invite a few players who are inside the large parties, then the other (many) players inside the large parties may reorganize the coalition structure to punish the deviating members; and (c) if they invite so many inside players that they exceed the large parties, then the members of the new party are too many to improve their power per capita.

Moreover, condition (ii) also suggests that the upper limit of difference $b_{\mathcal{P}}$ decreases as $|V T(\mathcal{P})|$ increases. Therefore, if a coalition structure contains three or more blocking coalitions, namely, a multiparty system, then the difference between the largest and smallest blocking coalitions has to be smaller for the structure to be stable, which yields multiple parties that are all very close and modest in size.

Condition (iii) shows that the configuration of $\mathcal{P}$ determines the lowest $\delta$ required for $\mathcal{P}$ to be stable. Note that the threshold $\frac{\log (|V T(\mathcal{P})|)}{\log \left(k /\left|S_{\mathcal{P}}^{\min }\right|\right)}$ lies between 0 and $1{ }^{* 3}$ One might have the following question: does the threshold admit Owen's $\delta(=1)$ and Penrose's $\delta(=0.5)$ ? For Owen's $\delta$, the answer is clear and affirmative. Since the threshold is less than or equal to one, it always admits Owen's $\delta$. Hence, all coalition structures pass (iii) if $\delta=1$. On the other hand, Penrose's $\delta$ is not as affirmative as Owen's. To see this, we make some calculations. For $\delta=0.5$, (iii) is equivalent to $k \geq|V T(\mathcal{P})|^{2} \cdot\left|S_{\mathcal{P}}^{\mathrm{min}}\right|$. From $|V T(\mathcal{P})| \geq 2$, it follows that $k \geq 4 \cdot\left|S_{\mathcal{P}}^{\min }\right|$, which suggests that the size of the smallest blocking coalition must be at most one-quarter of quota $k$. For such a coalition structure to exist, the quota $k$ has to be very high, and indeed, by $\left|S_{\mathcal{P}}^{\min }\right| \geq d$ and $d=n-k+1$, the preceding inequality $k \geq 4 \cdot\left|S_{\mathcal{P}}^{\min }\right|$ generates $k \geq \frac{4}{5}(n+1)$. In other words, more than approximately $80 \%$ of the voting members must be affirmative for a measure to pass. This quota is very high: for example, in most countries, the quota $k$ for constitutional amendment is chosen among $\frac{1}{2}+(50+\%), \frac{3}{5}(60 \%), \frac{2}{3}(66 \%)$ and $\frac{3}{4}(75 \%)$. In this sense, if $\delta$ is assumed to be 0.5 , we must set a high quota $k$ to obtain a partition satisfying the conditions.

We conclude this subsection by providing some technical remarks.

- The axioms used in Proposition 4.2 imply those in Proposition 4.1: $[\mathbf{E F} \#$ and $\mathbf{N P \# ~} \Rightarrow \mathbf{D}]$ and $[\mathbf{E T} \# \Rightarrow \mathbf{E T}-]$ and $[\mathbf{E F} \# \Rightarrow \mathbf{F}-]$.

- If $n$ is very small, the partition satisfying the conditions required in Proposition 4.2 may not exist:

\footnotetext{
${ }^{* 3}$ From (i) and the fact that $S_{\mathcal{P}}^{\min }$ is the smallest blocking coalition in $\mathcal{P}$, it follows that $k \geq|V T(\mathcal{P})| \cdot\left|S_{\mathcal{P}}^{\min }\right|$. Therefore, the threshold is at most one. It is more than zero because (i) implies that $|V T(\mathcal{P})| \geq 1$. It attains one if all blocking coalitions are identical in size, which means that $b_{\mathcal{P}}=0$. As $b_{\mathcal{P}}$ increases, the threshold decreases.
} 
for example, $n=3$ and $k=2$. The "smallest" example for which Proposition 4.2 holds is $n=5$, $k=4, \delta=1$ and $\psi=$ SS. Partition $[2,2,1]$ is an element of the pessimistic core.

- Abe (2018) shows that if $\psi=\mathrm{SS}, \delta=1$ and $k=H(n)$, then for every $n \geq 3$, a partition belongs to the pessimistic core if and only if the partition contains a minimal winning coalition.

- The minimal element of $\Lambda$ can switch even within the interval of $\delta$ satisfying (iii). The switch occurs because $k-1>d$ and $2<\left(n-a^{*}\right) / d$ for $|V T(\mathcal{P})| \geq 2$. Note that if $\delta=1$, then the right element of $\Lambda$ is smaller than the other, and we have $\Lambda^{n, k}(\delta)=n-a^{*}$ for every $n \geq 3$ and every $k$ with $H(n) \leq k \leq n$.

\subsection{The projective core}

The projective core is a subset of the pessimistic core and may be empty for some combination of $(n, k, \delta, \psi)$. The following result shows that even if a coalition structure contains a winning coalition, it can be excluded from the projective core. This result holds with the following undemanding axioms. We define $\Omega^{n}(\delta):=\frac{\sqrt[\delta]{2}}{1+\sqrt[\delta]{2}}(n+1)$ for $0<\delta \leq 1$, elaborated later in Figure 1 .

Proposition 4.3. Let $H(n) \leq k \leq n, 0<\delta \leq 1$ and $\mathcal{P} \in \Pi(N)$. Let $\psi$ satisfy D, ET-, EF2-. If $\Omega^{n}(\delta)<k \leq n$ and there is $S \in \mathcal{P}$ such that $s \geq k$, then $\mathcal{P} \notin C^{\operatorname{proj}}\left(\phi^{\psi,(n, k), \delta}\right)$.

Proposition 4.3 shows that if quota $k$ is so high that it exceeds $\Omega^{n}$, any winning coalition, including a minimal winning coalition, no longer helps the coalition structure lie in the projective core. As illustrated in Figure $1, \Omega^{n}(\delta)$ is a decreasing function with respect to $\delta$. We have $\Omega(1)=\frac{2}{3}(n+1)$ and $\lim _{\delta \rightarrow 0} \Omega(\delta)=$ $(n+1)$. Therefore, for $k$ to be in the interval $\left(\Omega^{n}(\delta), n\right], \delta$ must be assumed to be at $\operatorname{least} \frac{\log 2}{\log n}$. Hence, $\delta=1$ is an option that is always available for every $n \geq 3$ and maximizes the interval of $k$. Penrose's $\delta=0.5$ is also available for every $n \geq 4$ as $\frac{\log 2}{\log 4}=0.5$ and requires the higher and shorter interval than $\delta=1$ does. This observation suggests that Penrose's $\delta$ might be more favorable than $\delta=1$ for some coalition structures to be stable because, as Proposition 4.3 states, $\Omega^{n}(\delta)<k \leq n$ is a sufficient condition for a partition with a winning coalition not to be in the projective core. Therefore, a smaller $\delta$ shortens the interval of $k$, which excludes the partitions with a winning coalition from the projective core, and extends the other interval of $k$, which may admit them into the projective core.

Remark 4.4. The following examples should strengthen the observation that a high quota $k$ is unfavorable for coalition structures to be in the projective core. For simplicity, let $\delta=1$, and consider $k>\frac{2}{3}(n+1)=\omega^{n}(1)$. If $n=8$ and $k=7$, then we have the following strong result: $C^{\operatorname{proj}}\left(\phi^{\psi,(n, k), \delta}\right)=\emptyset$ for $\psi=\mathrm{SS}$, BZ, NBZ, DP. Moreover, if $n=9$ and $k=7$, then $C^{\operatorname{proj}}\left(\phi^{\psi,(n, k), \delta}\right)=\emptyset$ for $\psi=\mathrm{SS}, \mathrm{BZ}$, NBZ; if $k=8$, then $C^{\operatorname{proj}}\left(\phi^{\psi,(n, k), \delta}\right)=\emptyset$ for $\psi=\mathrm{SS}$, DP. These examples show that for a high quota $k$, the projective core tends to be empty for various power indices. In addition, the projective core is given as follows for each nonempty case: $C^{\operatorname{proj}}\left(\phi^{\mathrm{DP},(9,7), 1}\right)=\{[4,3,1,1]\}, C^{\operatorname{proj}}\left(\phi^{\mathrm{BZ},(9,8), 1}\right)=\{[4,4,1],[4,2,2,1]\}$ and $C^{\operatorname{proj}}\left(\phi^{\mathrm{NBZ},(9,8), 1}\right)=\{[2,2,2,2,1]\}$. These examples also suggest that even if some coalition structure belongs to the projective core with respect to some power index, it does not with respect to another power index. In this sense, it is not straightforward for a coalition structure to be in the projective core 


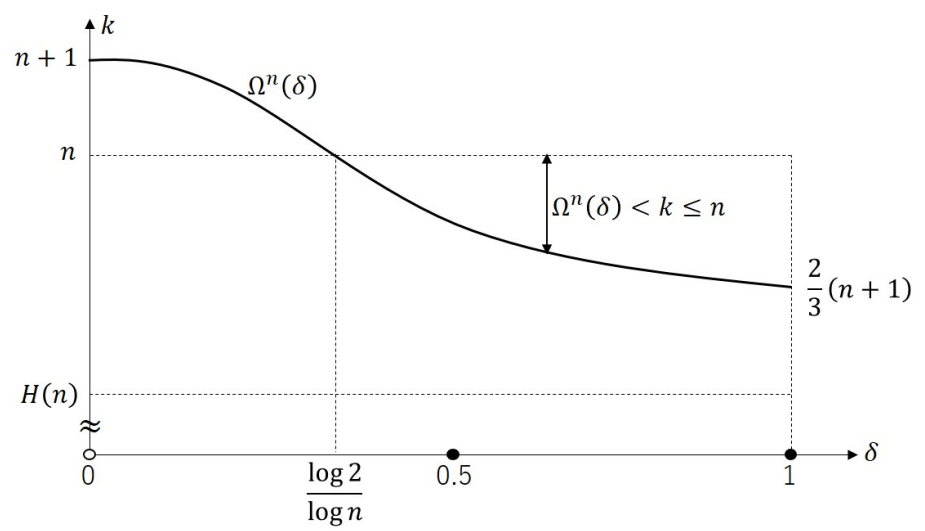

Figure 1 Function $\Omega^{n}$ and admissible quota $k$

for a wide range of power indices if $k$ is high. ${ }^{* 4}$

Proposition 4.3 and Remark 4.4 suggest that a high quota $k\left(>\Omega^{n}(\delta)\right)$ is unfavorable for many coalition structures to be stable in terms of the projective core. In contrast, the next proposition shows that a lower $k$ can be favorable. To see this, we need an additional axiom.

We first consider a weighted majority game that consists of a dictatorial party, which has $x$ votes, and $t$ one-vote individuals: $v_{1}=(k ; x, 1, \ldots, 1)$ with $x \geq k$. Now, we suppose that the dictatorial party collapses and splits into two parts, each of which is nondictatorial, formally, a party with $w_{1}$ votes and the other with $w_{2}$ votes, where $w_{1}+w_{2}=x \geq k, 1 \leq w_{1}<k$ and $1 \leq w_{2}<k$. This split generates the following weighted majority game $v_{2}=\left(k ; w_{1}, w_{2}, 1, \ldots, 1\right)$ with $t$ one-vote individuals. If a weighted majority game satisfies these three conditions above, we say that the game is a divided dictator game (a $d d$-game) with $t$ one-vote players. For convenience, even when $w_{1}$ or $w_{2}$ is equal to 1 , we say that the game has $t$ one-vote players and do not say that $t+1$ nor $t+2$ one-vote players. Note that $t$ can be zero, $t \geq 0$. The following property requires that a power index sets an upper limit for the nondictatorial parties. Let $k$ be an integer with $k \geq 2$.

Axiom 10. (Upper Limits of Divided Dictator for Quota $k, k$-UL)

Let $1 \leq w_{1}<k, 1 \leq w_{2}<k, k \leq w_{1}+w_{2}$ and $t \geq 0$. Let $v=\left(k ; w_{1}, w_{2}, 1, \ldots, 1\right)$ be a dd-game with $t$

\footnotetext{
${ }^{* 4}$ Some readers might be interested in the extreme case $k=n$. This can be thought of as a special voting system in which decisions are made by an affirmative vote of all voters, and in fact, the projective core for this case tends to be different from the other cases: for $\delta=1$, if $\psi$ satisfies EF- and ET-, then $\mathcal{P}=\{\{i\} \mid i \in N\}$ is the only element of the projective core. If $\psi=\mathrm{BZ}$, which violates EF-, then $n=6$ is a counterexample, for which the projective core is empty, and we have $[1,1,1,1,1,1] \notin C^{\text {proj }}$ because forming a three-person coalition improves their individual Banzhaf values, $1 / 24>1 / 32$.
} 
one-vote players. Power index $\psi$ obeys

$$
\psi_{i_{1}}(v) \leq \frac{w_{1}}{k} \text { and } \psi_{i_{2}}(v) \leq \frac{w_{2}}{k}
$$

where $i_{1}\left(i_{2}\right)$ is the player who has $w_{1}\left(w_{2}\right)$ votes in $v$.

Lemma 4.5 shows that for a low quota $k \leq \frac{2}{3}(n+1)$, the following two power indices obey this axiom, where $\frac{2}{3}(n+1)$ stands for the minimum of $\Omega^{n}: \frac{2}{3}(n+1)=\min _{\delta: \frac{\log 2}{\log n} \leq \delta \leq 1} \Omega^{n}(\delta)$.

Lemma 4.5. For every $k$ with $H(n) \leq k \leq \frac{2}{3}(n+1)$, the Shapley-Shubik power index and the DeeganPackel power index satisfy $k$-UL.

Although we omit the proof of this lemma since it is long and computational, we provide some counterexamples below. The Banzhaf power index and the normalized Banzhaf power index violate $k$-UL for some $k$ with $1 \leq k \leq \frac{2}{3}(n+1)$. For example, let $n=15$ and $k=10$, and consider dd-game $(10 ; 9,1,1,1,1,1,1)$, namely, $w_{1}=9$ and $w_{2}=1$. We obtain $\mathrm{BZ}_{i_{1}}=\frac{63}{64}>\frac{9}{10}$ and $\mathrm{NBZ}_{i_{1}}=\frac{63}{69}>\frac{9}{10}$, both of which violate the axiom. If $k>\frac{2}{3}(n+1)$, both Shapley-Shubik and Deegan-Packel power indices violate $k$-UL. For example, let $n=7$ and $k=6$, and consider dd-game $(6 ; 4,2,1)$, namely, $w_{1}=4$ and $w_{2}=2$. We have $\mathrm{SS}_{i_{2}}=\mathrm{DP}_{i_{2}}=\frac{1}{2}>\frac{2}{6}$. Therefore, the following proposition shows that this axiom is a common property to the Shapley-Shubik and Deegan-Packel power indices (and other nameless indices) that contributes to the projective core if $k$ is relatively low.

Proposition 4.6. Let $H(n) \leq k \leq \frac{2}{3}(n+1)$ and $0<\delta \leq 1$. If $\psi$ satisfies $\mathbf{D}$ and $k$-UL, then $C^{\operatorname{proj}}\left(\phi^{\psi,(n, k), \delta}\right)$ is nonempty.

A minimal winning coalition again plays an important role in ensuring the nonemptiness of the projective core. As mentioned in Proposition 4.1, if a partition contains a minimal winning coalition, then the partition belongs to the pessimistic core no matter what coalition structure is formed by the players outside the minimal winning coalition. On the other hand, for the projective core, the coalition structure of those outside players matters. To be more precise, containing a minimal winning coalition does not imply that the partition belongs to the projective core. For example, let $n=7, k=5, \delta=1$ and $\psi=$ SS. Partition $[5,1,1]$ is an (and the only) element of the projective core, while partition $[5,2]$ is not. Both $[5,1,1]$ and $[5,2]$ belong to the pessimistic core. What generates this difference? The notion of the casting vote is helpful to answer this question. Table 3 shows the distributions of voting power for each partition mentioned above. In partition [5,2], a three-person (sub)coalition in the five-person coalition has an incentive to deviate because this deviation generates partition $[3,2,2]$, in which the three-person coalition obtains the casting vote between the two two-person coalitions since the quota $k$ is 5 . This incentive is precisely exhibited in Table $3: \frac{2}{9}>\frac{1}{5}$. On the other hand, if the partition is $[5,1,1]$, then the same deviation does not give an incentive to the three players because the resulting partition is $[3,2,1,1]$, in which the three-person coalition does not have the casting vote. This difference yields the gap between $[5,2]$ and $[5,1,1]$ and shows how the coalition structure outside the winning coalition matters to the projective core. Therefore, an intuition is summarized as follows: as the coalition structure of the players outside the winning coalition becomes finer, deviating players are less likely to obtain the casting 
vote and to improve their power. Hence, the coalition structure becomes more stable in the sense of the projective core.

\begin{tabular}{|c|c|c|c|c|c|c|c|c|}
\hline Partition & & & $\operatorname{ting} \mathrm{p}$ & wer pe & capita & & & $C^{\text {proj }}$ \\
\hline$[5,2]$ & $1 / 5$ & $1 / 5$ & $1 / 5$ & $1 / 5$ & $1 / 5$ & 0 & 0 & \\
\hline$[5,1,1]$ & $1 / 5$ & $1 / 5$ & $1 / 5$ & $1 / 5$ & $1 / 5$ & 0 & 0 & + \\
\hline$[3,2,2]$ & $2 / 9$ & $2 / 9$ & $2 / 9$ & $1 / 12$ & $1 / 12$ & $1 / 12$ & $1 / 12$ & \\
\hline$[3,2,1,1]$ & $7 / 36$ & $7 / 36$ & $7 / 36$ & $1 / 8$ & $1 / 8$ & $1 / 12$ & $1 / 12$ & \\
\hline
\end{tabular}

\section{Conclusion}

We can derive the following messages about the stability of coalition structures from our results and analysis.

- If a coalition structure contains an over-winning coalition, namely, a winning coalition that is not minimal, then the coalition structure is not stable in the senses of the pessimistic core and the projective core because a minimal winning coalition has an incentive to deviate and wins "more efficiently" with fewer members.

- If a coalition structure contains a minimal winning coalition, then it tends to be stable: such a coalition structure belongs to the pessimistic core for every quota more than $50 \%$ and a wide range of power indices, while it fails to be in the projective core if the quota is high $\left(>\Omega^{n}\right)$. If the quota is low, there is a coalition structure that contains a minimal winning coalition and belongs to the projective core. However, the partial coalition structure outside the minimal winning coalition contributes to the entire coalition structure of all players being in the projective core.

- The pessimistic core can contain a coalition structure without any winning coalitions if the coalition structure satisfies some conditions. A two-party system is a typical example. The conditions require that the two large parties are close in size and that the other minor parties and independents are so small that they are not influential in the coalition structure.

We conclude this paper with a technical problem left open. The Banzhaf power index does not satisfy EF\#. For example, the Banzhaf power index assigns distribution $\left(\frac{1}{4}, \frac{1}{4}, \frac{1}{4}, 0\right)$ to weighted majority game $(8 ; 4,2,2,1)$, in which the first three players are veto players and the fourth player is a null player. However, this example violates condition (ii) of Proposition 4.2 with, for example, $\delta=1$ as we have $b_{\mathcal{P}}=2>\frac{1}{3} \cdot 4-2=\frac{1}{\sqrt[\delta]{|V T(\mathcal{P})|}} \Lambda^{n, k}(\delta)-\left|S_{\mathcal{P}}^{\min }\right|$. We conjecture that the following statement may hold: the Banzhaf power index is efficient for every weighted majority game induced from a partition satisfying (ii) of Proposition 4.2. If this conjecture is true (for every $\delta$ ), then we may admit the Banzhaf power index into Proposition 4.2. However, even if it is true, the extended version of efficiency is artificial and lacks normative meaning: further research and adjustment are needed. 


\section{Appendix}

In the proofs, when $n, k, \delta$ and $\psi$ are fixed, we simply write $w^{\mathcal{P}}:=w^{(n, k), \mathcal{P}}$ and $\phi_{j}(\mathcal{P}):=\phi_{j}^{\psi,(n, k), \delta}(\mathcal{P})$ for every $\mathcal{P} \in \Pi(N)$.

\section{Proof of Proposition 4.1}

Proof. We fix $k$ with $H(n) \leq k \leq n$ and $\delta$ with $0<\delta \leq 1$. It follows from $H(n) \leq k$ that $0 \leq n-k<$ $k \leq n$. Let $K \subseteq N$ be a coalition that consists of $k$ players, $|K|=k$. Fix a partition $\mathcal{P} \in \Pi(N)$ that contains $K$ as an element. By $\mathbf{D}$, we have $\phi_{j}(\mathcal{P})=1 / k^{\delta}$ for every $j \in K$, and $\phi_{j}(\mathcal{P})=0$ for every $j \in N \backslash K$.

We first consider $S^{\prime} \subseteq N$ with $1 \leq s^{\prime} \leq n-k$. Let $\mathcal{Q}=\left\{S^{\prime}, N \backslash S^{\prime}\right\}$. By $\mathbf{D}$ and $n-s^{\prime} \geq k$, $\phi_{j}(\mathcal{Q})=0 \leq \phi_{j}(\mathcal{P})$ for every $j \in S^{\prime}$. Therefore, $S^{\prime}$ does not pessimistically deviate from $\mathcal{P}$.

Now, we consider $S^{\prime} \subseteq N$ with $k \leq s^{\prime} \leq n$. By $k \leq s^{\prime}$ and $H(n) \leq k$, it holds that $S^{\prime} \cap K \neq \emptyset$. In the same manner as the preceding case, let $\mathcal{Q}=\left\{S^{\prime}, N \backslash S^{\prime}\right\}$. Now, by $\mathbf{D}$ and $k \leq s^{\prime}$, we obtain $\phi_{j}(\mathcal{Q})=1 / s^{\prime \delta} \leq 1 / k^{\delta}=\phi_{j}(\mathcal{P})$ for every $j \in S^{\prime} \cap K$, and $S^{\prime}$ does not pessimistically deviate form $\mathcal{P}$.

Next, let $n-k+1(=d) \leq s^{\prime} \leq k-1$. Let $m$ be the integer part of $\left(n-s^{\prime}\right) / d$ and $a$ be the remainder of $\left(n-s^{\prime}\right) / d$, namely,

$$
m \cdot d+a=n-s^{\prime},
$$

where $0 \leq a \leq d-1(=n-k)$. Note that $m \geq 1$ because we have $n-s^{\prime} \geq n-k+1=d$ by $s^{\prime} \leq k-1$. If $m=1$, define $\mathcal{Q}=\left\{S^{\prime}, T^{+}\right\}$, where $T^{+}=N \backslash S^{\prime}$ and $\left|T^{+}\right|=d+a$. If $m \geq 2$, define a partition $\mathcal{Q}=\left\{S^{\prime}, T^{+}, T_{1}, \ldots, T_{m-1}\right\}$ that satisfies $\left|T_{l}\right|=d$ for every $l=1, \ldots, m-1,\left|T^{+}\right|=d+a, T \cap T^{\prime}=\emptyset$ for every $T, T^{\prime} \in \mathcal{Q}$ with $T \neq T^{\prime}$, and $\cup_{T \in \mathcal{Q}} T=N$. Hence, for every $m \geq 1$, all elements $T \in Q$ satisfy $|T| \geq d$. In other words, all coalitions in $\mathcal{Q}$ are veto players in $w^{Q}$. Therefore, by ET- and F-, for every $j \in S^{\prime}$, we obtain $\phi_{j}(\mathcal{Q}) \leq 1 /\left((m+1) \cdot s^{\prime \delta}\right)$. Since $s^{\prime} \geq n-k+1, S^{\prime} \cap K \neq \emptyset$. Let $j \in S^{\prime} \cap K$. We have

$$
\phi_{j}(\mathcal{P})-\phi_{j}(\mathcal{Q}) \geq \frac{1}{k^{\delta}}-\frac{1}{(m+1) \cdot s^{\prime \delta}}=\frac{1}{k^{\delta}(m+1) s^{\prime \delta}}\left[(m+1) \cdot s^{\prime \delta}-k^{\delta}\right] .
$$

By (1), we have $m=\left(n-s^{\prime}-a\right) / d$. Hence,

$$
\begin{aligned}
(2) & =\frac{1}{k^{\delta}(m+1) s^{\prime} \delta}\left[\left(\frac{n-s^{\prime}-a}{d}+1\right) \cdot s^{\prime \delta}-k^{\delta}\right] \\
& =\frac{1}{k^{\delta}(m+1) s^{\prime}} \frac{1}{d}\left[\left((d+n-a)-s^{\prime}\right) s^{\prime \delta}-k^{\delta} d\right] .
\end{aligned}
$$

Below, we focus on the term inside the brackets

$$
\left((d+n-a)-s^{\prime}\right) s^{\delta}-k^{\delta} d
$$

and show that $(3) \geq 0$. Since $d \leq s^{\prime} \leq k-1$ and (3) is concave for $s^{\prime} \in[d, k-1]$, (3) achieves its minimum at either $s^{\prime}=d$ or $s^{\prime}=k-1$. If $s^{\prime}=d$, then $(3)=(n-a) d^{\delta}-k^{\delta} d$. By $a \leq n-k$, (3) $\geq d^{\delta} k-k^{\delta} d=k^{\delta} d^{\delta}\left(k^{1-\delta}-d^{1-\delta}\right) \geq 0$ as $k \geq d$. If $s^{\prime}=k-1$, then $n-s^{\prime}=d$, which implies $a=0$. Therefore, $(3)=d\left(2 \cdot(k-1)^{\delta}-k^{\delta}\right)$. We have $2 \geq(k /(k-1))^{\delta}$ as $k \geq 2$ for every $n \geq 3$. Note 
that $1<k /(k-1) \leq 2$ and $0<\delta \leq 1$. Thus, it follows that $(3) \geq 0$ for $s^{\prime}=k-1$, and we obtain $\phi_{j}(\mathcal{P})-\phi_{j}(\mathcal{Q}) \geq 0$.

\section{Proof of Proposition 4.2}

Proof. Let $\mathcal{P}$ and $\delta$ satisfy the conditions of the proposition. Below, for simplicity, we omit $\mathcal{P}$ and write $s^{\min }:=\left|S_{\mathcal{P}}^{\min }\right|, s^{\max }:=\left|S_{\mathcal{P}}^{\max }\right|$ and $b:=b_{\mathcal{P}}$. In addition, let $m:=|V T(\mathcal{P})|$. If $\mathcal{P} \backslash V T(\mathcal{P}) \neq \emptyset$, let $S^{*} \in \mathcal{P} \backslash V T(\mathcal{P})$. We consider an ordering $\sigma$ of players of $w^{\mathcal{P}}$ (hence, players are coalitions in $\mathcal{P}$ ). For the ordering $\sigma$, if there is $\hat{S} \in V T(\mathcal{P})$ such that $\hat{S}$ a successor of $S^{*}$ in $\sigma$, then $S^{*}$ is not the pivot of $\sigma$ as $\hat{S} \in V T(\mathcal{P})$ is a veto player in $w^{\mathcal{P}}$. If all $\hat{S} \in V T(\mathcal{P})$ are predecessors of $S^{*}$ in $\sigma$, then again $S^{*}$ is not the pivot of $\sigma$ because, in view of (i), we have $\left|\cup_{\hat{S} \in V T(\mathcal{P})} \hat{S}\right|=k$, and the union of the predecessors of $S^{*}$ is winning. Hence, if $\mathcal{P} \backslash V T(\mathcal{P}) \neq \emptyset$, every coalition $S \in \mathcal{P} \backslash V T(\mathcal{P})$ is a null player in $w^{\mathcal{P}}$. Therefore, by $\mathbf{E F} \#, \mathbf{E T} \#$ and NP\#, we have

$$
\phi_{i}(\mathcal{P})=\frac{1}{m \cdot s^{\delta}}
$$

for every $S \in V T(\mathcal{P})$ and every $i \in S$. Now, let $S^{\prime} \subseteq N$ be a nonempty coalition and denote $s^{\prime}:=\left|S^{\prime}\right|$.

If $1 \leq s^{\prime} \leq n-k$, let $\mathcal{Q}=\left\{S^{\prime}, N \backslash S^{\prime}\right\}$. By NP\# and $s^{\prime} \leq n-k, \phi_{j}(\mathcal{Q})=0 \leq \phi_{j}(\mathcal{P})$ for every $j \in S^{\prime}$. Hence, $S^{\prime}$ does not pessimistically deviate form $\mathcal{P}$.

If $k \leq s^{\prime} \leq n$, then $S^{\prime} \cap S \neq \emptyset$ for every $S \in V T(\mathcal{P})$, because if there is $S \in V T(\mathcal{P})$ such that $S^{\prime} \cap S=\emptyset$ then $\left|S^{\prime} \cup S\right|=\left|S^{\prime}\right|+|S| \geq k+(n-k+1)>n$ yields a contradiction. Let $i \in S^{\prime} \cap S^{\text {min }}$. By $s^{\prime} \geq k, \mathbf{E F} \#$ and NP\# imply that $\phi_{i}(\mathcal{Q})=1 / s^{\prime \delta}$ for every $Q \in \Pi(N)$ with $S^{\prime} \in \mathcal{Q}$. We fix an arbitrary $Q \in \Pi(N)$ with $S^{\prime} \in \mathcal{Q}$. Now, in view of (iii), we have $\delta \geq \frac{\log (m)}{\log \left(k / s^{\min }\right)}$. This is equivalent to $\log m \leq \delta\left(\log k-\log s^{\min }\right)$. Therefore, we have

$$
m \cdot\left(s^{\min }\right)^{\delta} \leq k^{\delta} .
$$

From $i \in S^{\prime} \cap S^{\text {min }},(4), s^{\prime} \geq k$ and (5), it follows that

$$
\phi_{i}(\mathcal{P})-\phi_{i}(\mathcal{Q})=\frac{1}{m \cdot\left(s^{\min }\right)^{\delta}}-\frac{1}{s^{\prime \delta}} \geq \frac{1}{m \cdot\left(s^{\min }\right)^{\delta}}-\frac{1}{k^{\delta}} \geq 0 .
$$

Thus $S^{\prime}$ does not pessimistically deviate form $\mathcal{P}$.

If $n-k+1(=d) \leq s^{\prime} \leq k-1$, then by (i), $S^{\prime} \cap S \neq \emptyset$ for some $S \in V T(\mathcal{P})$. For every $S \in V T(\mathcal{P})$, every $i \in S$ and every $j \in S^{\max }$, by $(4)$, it holds that $\phi_{i}(\mathcal{P})=1 /\left(m \cdot s^{\delta}\right) \geq 1 /\left(m \cdot\left(s^{\max }\right)^{\delta}\right)=\phi_{j}(\mathcal{P})$. Therefore, it suffices to show that $S^{\prime}$ with $S^{\prime} \cap S^{\max } \neq \emptyset$ does not pessimistically deviate from $\mathcal{P}$. Let $i \in S^{\prime} \cap S^{\max }$. Let $m^{\prime}$ be 1 plus the integer part of $\left(n-s^{\prime}\right) / d$, namely, $m^{\prime}=\left\lfloor\left(n-s^{\prime}\right) / d\right\rfloor+1$, where $m^{\prime} \geq 2$ since $s^{\prime} \leq k-1$ implies $n-s^{\prime} \geq d$. Let $a$ be the remainder of $\left(n-s^{\prime}\right) / d$. Hence $0 \leq a \leq d-1(=n-k)$. We obtain $n=s^{\prime}+\left(m^{\prime}-1\right) d+a$. Now, in a similar manner to the proof of Proposition 4.1, for $m^{\prime}=2$, let $\mathcal{Q}=\left\{S^{\prime}, T^{+}\right\}$, where $T^{+}=N \backslash S^{\prime}$ and $\left|T^{+}\right|=d+a$. For $m^{\prime} \geq 3$, define a partition $\mathcal{Q}=\left\{S^{\prime}, T^{+}, T_{1} \ldots, T_{m^{\prime}-2}\right\}$, where $\left|T_{l}\right|=d$ for $l=1, \ldots, m^{\prime}-1, T \cap T^{\prime}=\emptyset$ for every $T, T^{\prime} \in \mathcal{Q}$ with $T \neq T^{\prime}$, and $\cup_{T \in \mathcal{Q}} T=N$. By EF \# and ET\#, $\phi_{i}(\mathcal{Q})=1 /\left(m^{\prime} \cdot s^{\prime \delta}\right)$ for every $m^{\prime} \geq 2$. Hence, in view of $i \in S^{\prime} \cap S^{\max }, \phi_{i}(\mathcal{P})-\phi_{i}(\mathcal{Q}) \geq 0$ if and only if

$$
m^{\prime} \cdot s^{\prime \delta}-m \cdot\left(s^{\max }\right)^{\delta}
$$


is greater than or equal to 0 . Below, we prove this inequality. From $n=s^{\prime}+\left(m^{\prime}-1\right) d+a$, it follows that $m^{\prime}=\left((d+n-a)-s^{\prime}\right) / d$. Hence,

$$
(6)=\frac{1}{d}\left((d+n-a)-s^{\prime}\right) \cdot s^{\prime \delta}-m \cdot\left(s^{\max }\right)^{\delta} .
$$

In a similar manner to (3) in the proof of Proposition 4.1, (6) attains its minimum at either $s^{\prime}=d$ or $s^{\prime}=k-1$. If $s^{\prime}=d$, we have $(6)=\left(\left(n-a^{*}\right) / d\right) \cdot d^{\delta}-m \cdot\left(s^{\max }\right)^{\delta}$; and if $s^{\prime}=k-1$, we have $a=0$ and $(6)=2 \cdot(k-1)^{\delta}-m \cdot\left(s^{\max }\right)^{\delta}$. Now, in view of (ii), we have $b \leq \frac{1}{\sqrt[\delta]{m}} \Lambda^{n, k}(\delta)-s^{\min }$. This is equivalent to

$$
m \cdot\left(s^{\max }\right)^{\delta} \leq\left(\Lambda^{n, k}(\delta)\right)^{\delta}=\min \left\{2 \cdot(k-1)^{\delta}, \frac{n-a^{*}}{d} \cdot d^{\delta}\right\} .
$$

Hence, we obtain $(6) \geq \min \left\{2 \cdot(k-1)^{\delta}, \frac{n-a^{*}}{d} \cdot d^{\delta}\right\}-m \cdot\left(s^{\max }\right)^{\delta} \geq 0$. Thus, for the player $i \in S^{\prime} \cap S^{\max }$, $\phi_{i}(\mathcal{P})-\phi_{i}(\mathcal{Q}) \geq 0$ holds, and $S^{\prime}$ with $S^{\prime} \cap S^{\max } \neq \emptyset$ does not pessimistically deviate from $\mathcal{P}$.

\section{Proof of Proposition 4.3}

Proof. Suppose that $S \in \mathcal{P}$ satisfies $s \geq k$. From $k>\Omega^{n}(\delta)=\frac{\sqrt[\delta]{2}}{1+\sqrt[8]{2}}(n+1)$, it follows that $k>\sqrt[\delta]{2} \cdot d$. Consider $T \subseteq N$ that satisfies $t=d$ and $T \supsetneq N \backslash S$. Note that such a superset $T \supsetneq N \backslash S$ exists because $t=d=n-k+1 \geq n-s+1>n-s$. Hence, clearly $T \cap S \neq \emptyset$. Let $\mathcal{Q}=\{T\} \cup \mathcal{P}_{N \backslash T}=\{T, N \backslash T\}$, where $N \backslash T$ is a veto player in $w^{\mathcal{Q}}$ since $n-t=k-1>\sqrt[\delta]{2} \cdot d-1 \geq d$ : the last inequality holds because $\sqrt[\delta]{2} \geq 2$ for every $\delta \in(0,1]$ and $d \geq 1$. It is clear that $T$ is a veto player in $w^{\mathcal{Q}}$ as $t=d$. By ET- and EF2-, we have $\phi_{j}(\mathcal{Q})=1 /\left(2 \cdot t^{\delta}\right)$ for every $j \in T$. By $\mathbf{D}$, we obtain $\phi_{j}(\mathcal{P})=1 / s^{\delta}$ for every $j \in S$ and $\phi_{j}(\mathcal{P})=0$ for every $j \in N \backslash S$. Hence, for every $j \in T \cap S$, we obtain $\phi_{j}(\mathcal{Q})-\phi_{j}(\mathcal{P})=1 /\left(2 \cdot t^{\delta}\right)-1 / s^{\delta}=$ $\left(s^{\delta}-2 \cdot t^{\delta}\right) /\left(2 t^{\delta} s^{\delta}\right) \geq\left(k^{\delta}-2 \cdot t^{\delta}\right) /\left(2 t^{\delta} s^{\delta}\right)>0$, where the last inequality follows from $k>\sqrt[\delta]{2} \cdot d$ and $t=d$. Moreover, for every $j \in T \backslash S, \phi_{j}(\mathcal{Q})-\phi_{j}(\mathcal{P})=1 /\left(2 \cdot t^{\delta}\right)-0>0$. Thus, $T$ has an incentive to projectively deviate from $\mathcal{P}$.

\section{Proof of Proposition 4.6}

Proof. Let $K \subseteq N$ be a coalition that consists of $k$ players, $|K|=k$. Let $\mathcal{P}=\{K\} \cup\{\{i\} \mid i \in N \backslash K\}$. By $\mathbf{D}$, we have $\phi_{j}(\mathcal{P})=1 / k^{\delta}$ for every $j \in K$ and $\phi_{j}(\mathcal{P})=0$ for every $j \in N \backslash K$. Below, for every nonempty $S \subseteq N$, let $\mathcal{Q}=\{S\} \cup \mathcal{P}_{N \backslash S}$.

Consider $S \subseteq N$ with $s \geq k$. Since $k \geq H(n), S \cap K \neq \emptyset$. By D, we obtain $\phi_{j}(\mathcal{Q})=1 / s^{\delta} \leq 1 / k^{\delta}=$ $\phi_{j}(\mathcal{P})$ for every $j \in S \cap K$. Hence, $S$ does not projectively deviate from $\mathcal{P}$.

Now, consider $S \subseteq N \backslash K$. Since $K \in \mathcal{P}_{N \backslash S}, K$ is still an element of $\mathcal{Q}$. Hence, by $\mathbf{D}$, we have $\phi_{j}(\mathcal{Q})=0=\phi_{j}(\mathcal{P})$ for every $j \in S$, and $S$ does not projectively deviate from $\mathcal{P}$.

Next, consider $S \subseteq N$ and $s \leq k-1$ and $S \cap K \neq \emptyset$. From $s \leq k-1$, it follows that $K \backslash S \neq \emptyset$. Hence, we have $\mathcal{Q}=\{K \backslash S, S\} \cup\{\{i\} \mid i \in N \backslash(K \cup S)\}$, where $N \backslash(K \cup S)$ can be empty. Since $K \backslash S \neq \emptyset$ and $S \cap K \neq \emptyset$, we have $1 \leq s \leq k-1$ and $1 \leq|K \backslash S| \leq k-1$. Moreover, clearly $|S \cup K| \geq k$. Hence, $w^{\mathcal{Q}}$ is a dd-game with $|N \backslash(K \cup S)|$ one-vote players. By $k$-UL, for every $j \in S \cap K$, 
$\phi_{j}(\mathcal{Q}) \leq(s / k)\left(1 / s^{\delta}\right)=s^{1-\delta} \cdot k^{-1} \leq k^{1-\delta} \cdot k^{-1}=1 / k^{\delta}=\phi_{j}(\mathcal{P})$. Thus, $S$ does not projectively deviate from $\mathcal{P}$.

\section{References}

[1] Abe T and Funaki Y (2017) The Non-Emptiness of the Core of a Partition Function Form Game, International Journal of Game Theory 46: 715-736

[2] Abe T (2018) Stable coalition structures in symmetric majority games: A coincidence between myopia and farsightedness. Theory and Decision 85: 353-374

[3] Bloch F and van den Nouweland A (2014) Expectation formation rules and the core of partition function games. Games and Economic Behavior 88: 339-353

[4] van den Brink R and van der Laan G (1998) Axiomatizations of the normalized Banzhaf value and the Shapley value. Social Choice and Welfare 15: 567-582

[5] Chwe MSY (1994) Farsighted coalitional stability. Journal of Economic Theory 63: 299-325

[6] Funaki Y and Yamato T (1999) The core of an economy with a common pool resource: A partition function form approach. International Journal of Game Theory 28: 157-171

[7] Shapley LS and Shubik M (1954) A method for evaluating the distribution of power in a committee system. American Political Science Review 48: 787-792

[8] Banzhaf JF (1965) Weighted voting doesn't work: A mathematical analysis. Rutgers Law Rev 19: 317-343

[9] Deegan J and Packel EW (1978) A new index of power for simple n-person games. International Journal of Game Theory 7: 113-123

[10] Hart S and Kurz M (1983) Endogenous formation of coalitions. Econometrica 51: 1047-1064

[11] Hart S and Kurz M (1984) Stable coalition structures, in Coalitions and Collective Action (MJ Holler, Ed.). Wüerzburg, Physica-Verlag

[12] Kóczy L (2007) A recursive core for partition function form games. Theory and Decision 63: 41-51

[13] Owen G (1977) Values of games with a priori unions, in Essays in Mathematical Economics and Game Theory (R Henn and O Moeschlin, Eds.). New York, Springer-Verlag

[14] Penrose LS (1946) The elementary statistics of majority voting. Journal of the Royal Statistical Society 109: 53-57

[15] Xue L (1997) Nonemptiness of the largest consistent set. Journal of Economic Theory 73: 453-459 